\title{
Ecological Assessment of Organochlorine Pesticides in the Surface Water from Huaihe River, China
}

\author{
Chunnian Da, Mei Yue, and Shengping Liu
}

\begin{abstract}
Thirty-four surface water samples were collected from Huaihe River, China. The organic compounds were extracted by solid-phase extraction (SPE) system and detected by gas chromatography (GC) using a mass-selective detector. HCHs were the dominant organochlorine pesticides in surface water. The results of PCA suggested there was some similar interrelation between individual OCPs. Health risks assessment indicated that the risks of children were higher than adults both carcinogenic risk and non-carcinogenic risk. The carcinogenic risk analysis indicated the health risks were very low for children, and there were not carcinogenic risks for adult.
\end{abstract}

Index Terms-HCH, DDT, health risk assessment, Huaihe River.

\section{INTRODUCTION}

Organochlorine pesticides (OCPs), which are typical POPs, have caused worldwide concern due to their high toxicity, persistence, semi-volatility, and bioconcentration [1], [2]. They can get into the aquatic environment through effluent discharge, agricultural runoff and atmospheric deposition. Information on OCPs concentrations in aquatic water and the importance of evaluating human exposure have been reported in many lakes and river, such as, Poyang lake, Chaohu Lake, Honghu Lake and Yangtze River [3], [4]. To our knowledge, there are few studies on the assessment of OCPs in water from the Huaihe River. Huaihe River, one of the seven largest rivers in China, flows through five provinces of Henan, Hubei, Anhui, Jiangsu and Shandong. The river has become more and more vulnerable to water pollution due to increasing discharges of industrial and agriculture waste along with rapid development of the local economy [5]. This study aims to carry out a survey of OCPs in water from the Huaihe River, China. The levels of pollutants have been determined, and the environmental quality and the impact of prior usage of OCPs in the agricultural and industrial would be assessed.

\section{MAterials AND Methods}

Manuscript received April 24, 2018; revised July 20, 2018.

Chunnian Da is with Department of Biology \& Environment Engineering, Hefei University, Hefei, Anhui 230022, PR China. She is also with Key Laboratory for Ecological Environment in Coastal Areas, National Marine Environment Monitoring Center, Dalian, 116023, China (e-mail: 13855165692@163.com).

Mei Yue and Shengping Liu was with Key Laboratory for Ecological Environment in Coastal Areas, National Marine Environment Monitoring Center, Dalian, 116023, China.

\section{A. Sampling}

A total of thirty-four surface water samples were collected in July 2015. Fig. 1 showed the map of sampling locations. 5 $\mathrm{L}$ water was collected from each sampling site, and transported to the laboratory and kept at $-20{ }^{\circ} \mathrm{C}$ in the refrigerator until further study.

\section{B. Sample Preparation and Extraction}

1L water sample was leached via glass fiber, using a vacuum pump. 4, 4'-dichlorobiphenyl was added to the water as a recovery indicator. The organic compounds in each water sample were extracted by solid-phase extraction (SPE) system. Before the extraction, the SPE cartridges were first washed with $5 \mathrm{ml}$ dichloromethane, $5 \mathrm{ml}$ methanol and $5 \mathrm{ml}$ ultrapure water. The cartridges could not be dried when the sample was loaded. About $5 \mathrm{~g}$ anhydrous sodium sulfate was connected every cartridge. The water sample was extracted with $10 \mathrm{ml}$ dichloromethane solution for each elution, and every sample was eluted three times. The extracts were concentrated to $1 \mathrm{ml}$ with a rotary evaporator. $10 \mathrm{~mL}$ hexane was added as a change solvent, and the extracts were again concentrated to $1 \mathrm{~mL}$ and transferred to vial until analysis.

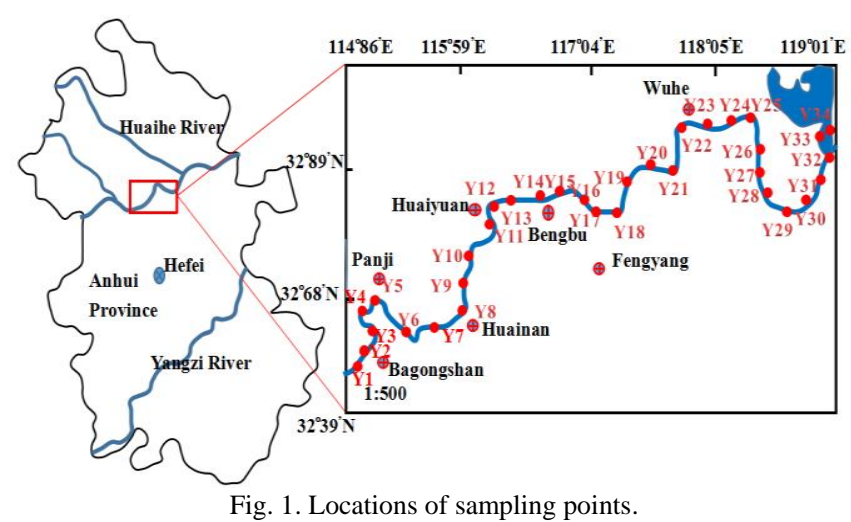

\section{Instrumental Analysis}

Sample analysis was conducted on an Agilent 6890 Series GC System connected to an Agilent 5973 Network Mass Selective Detector. A DB-5 MS fused silica capillary column $(30 \mathrm{~m} \times 0.25 \mathrm{~mm} \times 0.25 \mathrm{~mm})$ was used for separation. Helium gas $(99.999 \%)$ was used as the carrier gas. The samples $(1 \mu \mathrm{L})$ were injected automatically by splitless mode. The MS was operated in $\mathrm{EI}^{+}$mode with selected ion. Electron energy was $70 \mathrm{eV}$. The column flow was maintained at $1 \mathrm{~mL} \mathrm{~min}^{-1}$. The temperature change was programmed as follows: began at $80^{\circ} \mathrm{C}$ for $1 \mathrm{~min}$, ramping at a rate of $12^{\circ} \mathrm{C} \mathrm{min}^{-1}$ to $200{ }^{\circ} \mathrm{C}$ for $10 \mathrm{~min}$, then to $220^{\circ} \mathrm{C}$ at $1{ }^{\circ} \mathrm{C} \mathrm{min}{ }^{-1}$ and kept for $5 \mathrm{~min}$ and finally to $290{ }^{\circ} \mathrm{C}$ at $15^{\circ} \mathrm{C} \mathrm{min}{ }^{-1}$ withholding for $5 \mathrm{~min}$. 


\section{RESULTS AND DISCUSSION}

\section{A. Concentration Levels of Organochlorine Pesticides in} Surface Water

Concentrations of HCHs and DDTs in surface water are shown in Table I. The detection frequencies for the HCHs and DDTs are $87.5 \%$ and $21.56 \%$, respectively, indicating a wide event of $\mathrm{HCHs}$ in surface water from the Huaihe River. The average values of $\Sigma$ HCHs and DDTs are $13.71 \mathrm{ng} / \mathrm{L}$ and $0.077 \mathrm{ng} / \mathrm{L}$, respectively. The concentrations of $\mathrm{HCH}$ s were much higher than those of the DDTs. Overall; HCHs was the dominant organochlorine pesticides in water samples. Compared with OCPs in the sediments, in our previous study [6], the detection frequencies and the average values of the $\mathrm{HCHs}$ increased, however, the detection frequencies and the average values of the DDTs decreased. This is explained by that fact that DDTs have lower water solubility, lower vapour pressure and higher lipophilicity [7]; thus, DDTs were easier to remain in the particulate phase than HCHs [8]. In addition, the amount of HCHs used to be significantly larger than that of DDTs in the history of China [9]. Also, HCHs were banned a little later than DDTs in this study area.

\section{B. Inter-relation between Individual OCPS}

PCA is used to explore the relationship of the measured parameters and contribution of every contaminant among all the contaminants [10]. The results of PCA were presented in Fig 2. The three principal components explained $59.02 \%$ of the total variance. The high positive loadings of $\alpha-\mathrm{HCH}, \beta$ $\mathrm{HCH}, \gamma-\mathrm{HCH}$ and o'p-DDE in the same group of PC1 $(22.26 \%)$ recommend the same origin of these pesticides. PC2 accounting for $21.80 \%$ of the total variability was mainly related with p'p-DDE, o'p-DDT, o'p-DDD. These pesticides residues in the same group of PC2 could suggest the event status of them in the coastal areas. PC2 reflected the presence of these historical inputs of DDT and its degradation products in this area. As for PC3 (14.97\%), the loadings of $\delta$ - $\mathrm{HCH}$ and p'p-DDD suggest these pesticides could have similar migration characteristics.

TABLE I: CONCENTRATION OF OCPS (NG/L) IN SURFACE WATER

\begin{tabular}{|c|c|c|c|c|c|c|c|c|c|c|c|c|}
\hline $\begin{array}{c}\text { Sample } \\
\text { code }\end{array}$ & $\alpha-\mathrm{HCH}$ & $\beta-\mathrm{HCH}$ & $\gamma-\mathrm{HCH}$ & $\delta-\mathrm{HCH}$ & $\sum \mathrm{HCH}$ & o'p-DDE & p'p-DDE & o'p-DDT & p'p-DDT & p'p-DDD & o'p-DDD & $\sum \mathrm{DDT}$ \\
\hline $\mathrm{S} 1$ & 2.12 & 5.07 & 1.05 & 0.03 & 8.27 & n.d. & 0.04 & n.d. & n.d. & n.d. & n.d. & 0.04 \\
\hline $\mathrm{S} 2$ & 5.73 & 24.39 & 11.32 & n.d. & 41.44 & 0.12 & n.d. & n.d. & 0.013 & n.d. & n.d. & 0.133 \\
\hline $\mathrm{S} 3$ & 5.18 & 13.02 & 4.13 & n.d. & 22.33 & n.d. & n.d. & n.d. & n.d. & 0.19 & n.d. & 0.19 \\
\hline $\mathrm{S} 4$ & 2.12 & 11.28 & 3.21 & 0.12 & 16.73 & n.d. & n.d. & n.d. & n.d. & n.d. & n.d. & 0 \\
\hline S5 & 1.79 & 15.26 & 2.85 & 0.06 & 19.96 & n.d. & n.d. & n.d. & 0.163 & n.d. & n.d. & 0.163 \\
\hline S6 & 1.02 & 4.72 & 1.76 & n.d. & 7.5 & n.d. & 0.011 & 0.11 & n.d. & n.d. & n.d. & 0.121 \\
\hline S7 & 1.11 & 5.54 & 2.36 & n.d. & 9.01 & n.d. & n.d. & n.d. & n.d. & n.d. & n.d. & 0 \\
\hline S8 & 1.43 & 11.20 & 2.16 & 0.22 & 15.01 & n.d. & n.d. & n.d & n.d & n.d & n.d. & 0 \\
\hline S9 & 1.34 & 5.43 & 1.85 & 0.08 & 8.7 & n.d. & n.d. & n.d. & n.d & n.d. & 0.011 & 0.011 \\
\hline $\mathrm{S} 10$ & 1.91 & 13.9 & 2.87 & 0.09 & 18.77 & n.d. & n.d. & n.d. & n.d. & n.d. & n.d. & 0 \\
\hline S11 & n.d. & 12.99 & 2.67 & n.d. & 15.66 & 0.012 & n.d. & n.d & n.d. & 0.013 & n.d. & 0.013 \\
\hline $\mathrm{S} 12$ & 1.34 & 5.5 & 1.81 & 0.16 & 8.81 & 0.041 & n.d. & n.d. & n.d. & n.d. & n.d. & 0 \\
\hline S13 & 1.67 & 5.64 & 2.74 & 0.11 & 10.16 & n.d. & n.d. & n.d. & n.d. & 0.013 & n.d. & 0.013 \\
\hline S14 & 1.88 & 3.91 & 2.36 & 0.20 & 8.35 & n.d. & 0.013 & n.d. & n.d. & n.d. & n.d. & 0.013 \\
\hline S15 & 1.45 & 3.53 & 2.56 & n.d. & 7.54 & n.d. & 0.03 & n.d. & 0.12 & n.d. & 0.02 & 0.17 \\
\hline S16 & 0.94 & 3.09 & 1.87 & n.d. & 5.9 & n.d. & 0.25 & 0.11 & n.d. & n.d. & 0.12 & 0.48 \\
\hline S17 & 1.22 & 5.07 & 2.15 & 0.13 & 8.57 & 0.13 & n.d. & 0.04 & 0.09 & n.d. & 0.05 & 0.18 \\
\hline S18 & n.d. & 4.39 & 1.32 & n.d. & 5.71 & n.d. & n.d. & n.d. & 0.06 & n.d. & n.d. & 0.06 \\
\hline S19 & 0.28 & 3.02 & 1.13 & n.d. & 4.43 & 0.014 & n.d. & 0.05 & n.d. & n.d. & n.d. & 0.05 \\
\hline $\mathrm{S} 20$ & 1.12 & 11.28 & 2.11 & 0.12 & 14.63 & n.d. & n.d. & n.d. & n.d. & n.d. & 0.012 & 0.012 \\
\hline $\mathrm{S} 21$ & 1.19 & 10.16 & 1.85 & 0.05 & 13.25 & n.d. & n.d. & 0.03 & 0.013 & n.d. & n.d. & 0.043 \\
\hline $\mathrm{S} 22$ & 2.43 & 4.12 & 2.79 & n.d. & 9.34 & n.d. & 0.013 & 0.15 & n.d. & n.d. & n.d. & 0.163 \\
\hline $\mathrm{S} 23$ & 3.18 & 3.59 & 4.36 & n.d. & 11.13 & n.d. & n.d. & n.d. & n.d. & n.d. & n.d. & 0 \\
\hline S24 & 3.43 & 10.45 & 3.19 & 0.13 & 17.2 & n.d. & n.d. & 0.012 & n.d. & 0.31 & n.d. & 0.322 \\
\hline $\mathrm{S} 25$ & 5.54 & 6.43 & 1.95 & 0.01 & 13.93 & n.d. & n.d. & n.d. & 0.02 & n.d. & 0.01 & 0.03 \\
\hline S26 & 2.91 & 13.9 & 2.57 & 0.03 & 19.41 & n.d. & n.d. & n.d. & n.d. & 0.017 & n.d. & 0.017 \\
\hline S27 & 2.11 & 11.98 & 2.67 & n.d. & 16.76 & n.d. & n.d. & 0.01 & n.d. & 0.013 & n.d. & 0.023 \\
\hline $\mathrm{S} 28$ & 2.34 & 5.56 & 1.91 & 0.06 & 9.87 & n.d. & n.d. & n.d. & n.d. & n.d. & n.d. & 0 \\
\hline S29 & 0.67 & 4.64 & 2.07 & 0.13 & 7.51 & n.d. & n.d. & n.d. & n.d. & 0.019 & n.d. & 0.019 \\
\hline
\end{tabular}




\begin{tabular}{lllllllllllll}
\hline S30 & 2.88 & 13.91 & 2.96 & 0.26 & 20.01 & n.d. & 0.008 & n.d. & n.d. & n.d. & 0.001 & 0.009 \\
S31 & 3.45 & 3.53 & 4.56 & n.d. & 11.54 & 0.06 & n.d. & n.d. & n.d. & n.d. & 0.02 & 0.02 \\
S32 & 1.94 & 2.89 & 2.47 & n.d. & 7.3 & n.d. & n.d. & 0.11 & n.d. & n.d. & n.d. & 0.11 \\
S33 & 1.02 & 15.07 & 8.05 & 0.92 & 25.06 & n.d. & 0.01 & n.d. & n.d. & 0.13 & 0.05 & 0.19 \\
S34 & 4.73 & 20.39 & 1.32 & n.d. & 26.44 & n.d. & n.d. & n.d. & 0.01 & n.d. & n.d. & 0.01 \\
\hline
\end{tabular}

\section{Health Risks Assessment}

\section{Health risks assessment model}

Exposure routes of OCPs are the food chain, drinking water and steam absorption. This study uses a method which was proposed by USEPA [11] to calculate the exposure dose. Briefly, the exposed population is divided into two groups of children (0-6 years old) and adult (> 18 years old), the calculation formulas are as follows:

$$
\begin{gathered}
\mathrm{CDI}=(\mathrm{C} \times \mathrm{IR} \times \mathrm{EF} \times \mathrm{ED}) /(\mathrm{BW} \times \mathrm{AT}) \\
\mathrm{R}=\mathrm{CDI} \times \mathrm{SF}
\end{gathered}
$$$$
\mathrm{HI}=\mathrm{CDI} / \mathrm{RfD}
$$

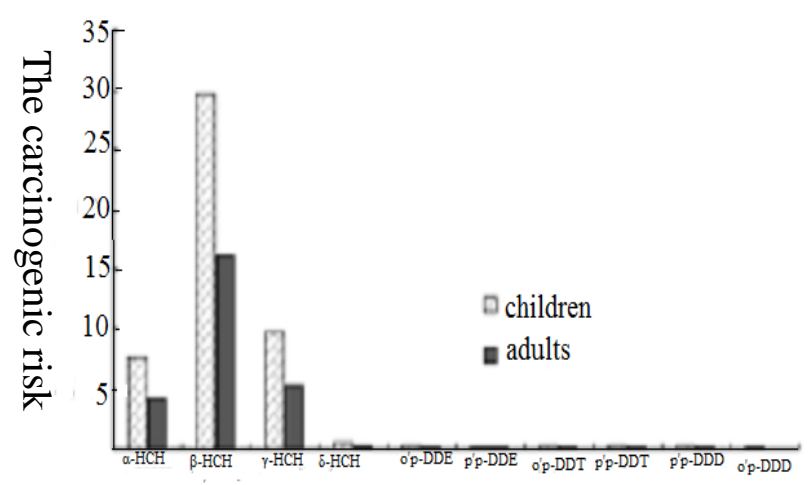

OCPs

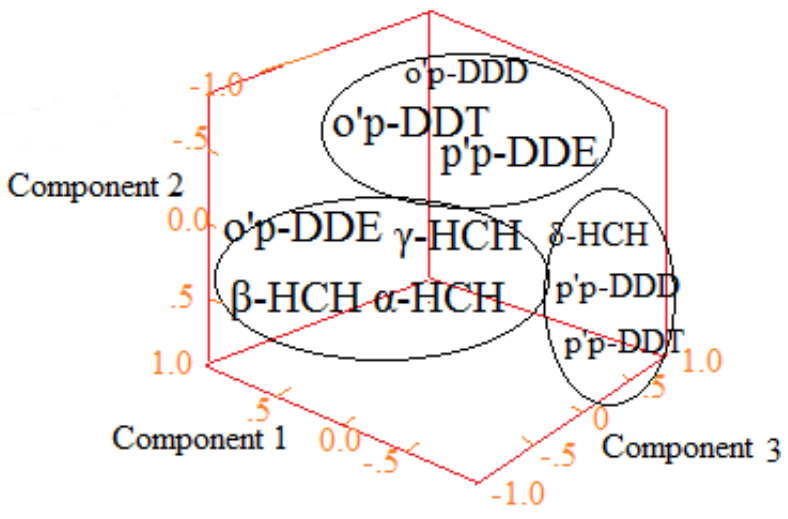

Fig. 2. Principal component analysis for OCPs.

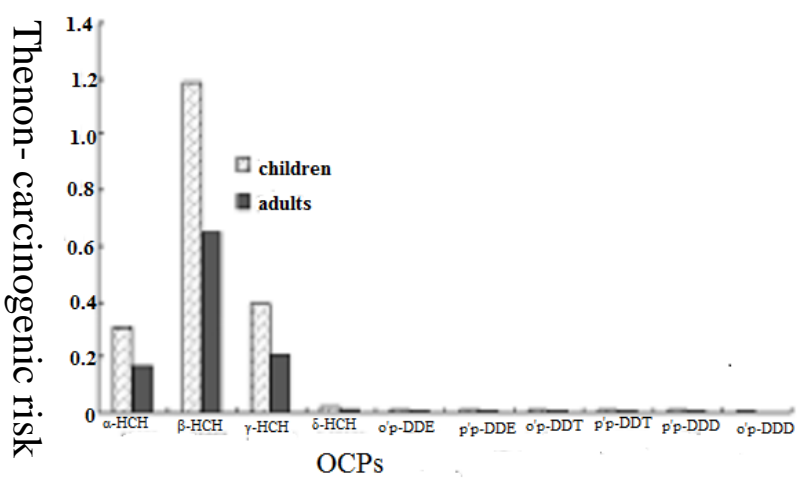

Fig. 3. Thcarcinogenic/non- carcinogenic risk $\left(\times 10^{-6}\right)$ for OCPs.

$\mathrm{CDI}$ is exposure dose of long time; $\mathrm{C}$ is the concentration of OCPs ( $\left.\mathrm{mg} \mathrm{L}^{-1}\right)$; IR is daily drinking dose (children: $1 \mathrm{~L}$ $\mathrm{d}^{-1}$, adult: $2.2 \mathrm{~L} \mathrm{~d}^{-1}$ );EF is the exposure frequency (350 d $\mathrm{a}^{-1}$ );ED is the exposure duration (children: $6 \mathrm{a}$, adult: 76.27 a); $\mathrm{BW}$ is the average body weight (children: $14 \mathrm{~kg}$, adult: 60 $\mathrm{kg}$ );AT is the averaging time (children: $2190 \mathrm{~d}$, adult: 26280 $\mathrm{d})$; $\mathrm{R}$ is the carcinogenic risk; $\mathrm{SF}$ is cancer slope factor $(2 \mathrm{~kg} \mathrm{~d}$ $\left.\mathrm{mg}^{-1}\right) ; \mathrm{HI}$ is the noncarcinogenic risk ; RfD is Reference Dose $\left(0.02 \mathrm{mg} \mathrm{kg}^{-1} \mathrm{~d}^{-1}\right)$;

\section{Health risks assessment}

According the above equation (2), (3), (4), we calculated annual averaged carcinogenic/noncarcinogenic risk caused by OCPs in surface water from the Huaihe River. The risk of the different compounds was showed in Table 4. The carcinogenic risk and non-carcinogenic risk of adults and children were showed in Fig. 3. As seen from the Fig .3, the risks of children were higher than adults both carcinogenic risk and non-carcinogenic risk. The results were consistent with the previous study [12, [13].

\section{ACKNOWLEDGMENTS}

This work is supported by Anhui Provincial Natural Science Foundation (1608085MD78), Key projects of Natural Science Research of universities in Anhui Province (KJ2015A201), Key Laboratory for Ecological Environment in Coastal Areas (201802), Key projects of Anhui province university outstanding youth talent support program (gxyqZD2016274), Special thanks are given to anonymous reviewers for their useful suggestions and comments.

\section{REFERENCES}

[1] Z. L. Zhang et al., "Fate and assessment of persistent organic pollutants in water and sediment from Minjiang River Estuary, Southeast China," Chemosphere, vol. 52, no. 9, pp. 1423-1430, 2003.

[2] C. Wu et al., "Risks from sediments contaminated with organochlorine pesticides in Hangzhou, China," Chemosphere, vol. 90, no. 9, 2341-2346, 2013.

[3] C. N. Da et al., "Distribution, sources, and ecological risks of organochlorine pesticides in surface sediments from the Huaihe River, China," Environ.Sci. Processes \& Impacts, vol. 15, no. 12, pp. 2288-2296, 2013.

[4] W. X. Liu et al., "The residues, distribution, and partition of organochlorine pesticides in the water, suspended solids, and sediments from a large Chinese lake (Lake Chaohu) during the high water level period," Environ. Sci.Pollut. R., vol. 20, no. 4, pp. 2033-2045, 2013. 
[5] C. Liu et al., "Levels of organochlorine pesticides in natural water along the Yangtze River, from headstream to estuary, and factors determining these levels," Environ. Earth Sci., vol. 62, no. 5, pp. 953-960, 2011

[6] J. Feng et al., "Residues of organochlorine pesticides (OCPs) in upper reach of the Huaihe River, East China," Ecotox. and environ. Safe, vol. 74 , no. 8, pp. 2252-2259, 2011.

[7] A. O. Barakat et al., "Persistent organochlorine pesticide and PCB residues in surface sediments of Lake Qarun, a protected area of Egypt," Chemosphere, vol. 90, no. 9, pp. 2467-2476, 2012.

[8] C. N. Da et al., "Analysis of HCHs and DDTs in a sediment core from the Old Huaihe River, China," Ecotox. Environ. Safe, vol. 100, pp. 171-177, 2014.

[9] A. E. Nemr et al., "Levels, distribution, and risk assessment of organochlorines in surficial sediments of the Red Sea coast, Egypt," Environ. Monit. Assess., vol. 185, no. 6, pp. 4835-4853, 2013.

[10] H. Hellar-Kihampa et al., "Spatial monitoring of organohalogen compounds in surface water and sediments of a rural-urban river basin in Tanzania," Sci. Total Environ., vol. 447, pp. 186-197, 2013.

[11] Y. Hu et al., "Distribution and health risk assessment of polychlorinated biphenyls in underground rivers of Chongqing, Southwest China," Acta Sci. Circumstantiae (in Chinese), vol. 31, pp. 1685-1690, 2011.

[12] L. Hu et al., "Occurrence and distribution of organochlorine pesticides (OCPs) in surface sediments of the Bohai Sea, China," Chemosphere, vol. 77, no. 5, pp. 663-672, 2009.

[13] I. Javedankherad et al., "Levels and distribution of organochlorine pesticides and polychlorinated biphenyls in water and sediment from the international Anzali Wetland, north of Iran," Bull. Environ.Contam. Toxicol, vol. 90, no. 3, pp. 285-290, 2013.

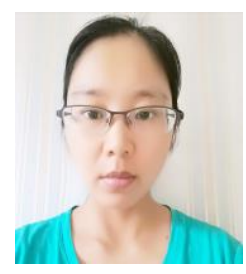

Chunnian Da was born on Aug 7, 1979, Anhui province, China. She got the doctor of science, persistent organic pollutants, University of Science \& Technology China, Hefei, China in 2014.

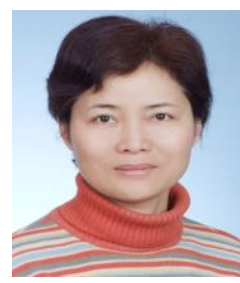

Mei Yue was born in Anhui province, China. She got the doctor of science, persistent organic pollutants, University of Mining and Technology China, Beijing, China in 2004.

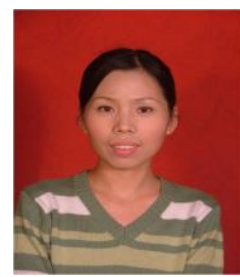

Shengping Liu was born in Anhui Province, China. She got the doctor of science, persistent organic pollutants, Hefei Polytechnic University, Hefei, China in 2006. 